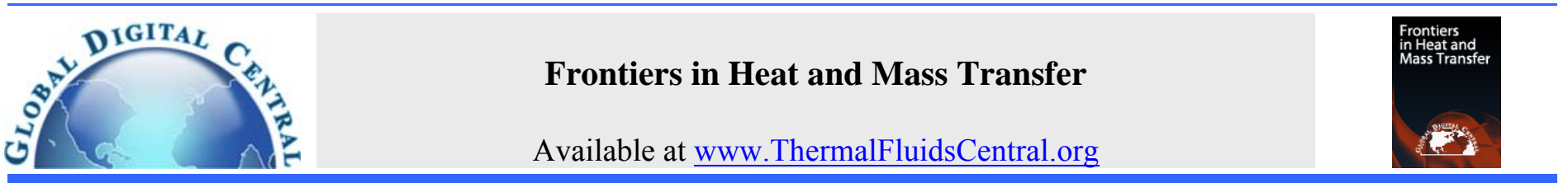

\title{
MHD FREE CONVECTIVE FLOW PAST AN IMPULSIVELY MOVING VERTICAL PLATE WITH RAMPED HEAT FLUX THROUGH POROUS MEDIUM IN THE PRESENCE OF INCLINED MAGNETIC FIELD
}

\author{
G. S. Seth ${ }^{\mathrm{a},}{ }^{*}$, P. K. Mandal ${ }^{\mathrm{a}}$ and A. J. Chamkha ${ }^{\mathrm{b}}$ \\ ${ }^{a}$ Department of Applied Mathematics, Indian Institute of Technology (ISM) Dhanbad - 826004, India \\ ${ }^{b}$ Mechanical Engineering Department, Prince Mohammad Bin Fahd University, Al-Khobar-31952, Kingdom of Saudi Arabia
}

\begin{abstract}
A theoretical investigation of unsteady hydromagnetic free convection flow with heat and mass transfer of a viscous, incompressible, electrically conducting, optically thick radiating and chemically reactive fluid near an impulsively moving vertical plate with ramped heat flux through fluid saturated porous medium in the presence of inclined magnetic field is carried out. Exact solutions of the governing equations for fluid velocity, fluid temperature and species concentration are obtained by Laplace transform technique. The expressions for the skin-friction, rate of mass transfer at the plate and plate temperature are also derived. Numerical results for fluid velocity, fluid temperature and species concentration are displayed graphically whereas those of skin-friction, rate of mass transfer at the plate and plate temperature are presented in tabular form for various values of pertinent flow parameters. It is found that fluid flow is accelerated / retarded by varying the angle of inclination of magnetic field.
\end{abstract}

Keywords: Free convection, inclined magnetic field, porous medium, ramped heat flux, chemical reaction.

\section{INTRODUCTION}

The phenomena of free convection arise in the fluid flow when temperature or concentration change causes density variation leading to buoyancy forces acting on the fluid elements. Natural convective flow is prevalent in many natural phenomena and has varied and wide range industrial applications. It plays a vital role in our environment. This can be seen in our everyday life in the atmospheric flow, which is driven by temperature or concentration differences. Natural processes such as vaporization of mist and fog, photosynthesis, drying of porous solids, transpiration, sea-wind formation and formation of ocean currents occur due to thermal and solutal buoyancy forces developed as a result of a difference in temperature or concentration or a combination of these two. Such configurations are also encountered in several practical systems for industry-based applications, viz., heat exchanger devices, cooling of molten metals, insulation systems, petroleum reservoirs, filtrations, chemical catalytic reactors and processes, nuclear waste repositories, desert coolers and wet bulb thermometers. Literature related to natural convection boundary layer flow with heat and mass transfer over various geometrical bodies is well documented by Eckert and Drake (1972), Kays and Crawford (1993), Nield and Bejan (2006) and Incropera et al. (2011).

Moreover, studies on MHD natural convection flow with simultaneous effect of heat and mass transfer in porous and non-porous media are encountered in extraction of oil through porous rocks, in the filtration of liquid metals, drag permeation through human skin, heat removal from nuclear debris, boundary layer flow control, prevention of scaling in heat exchangers, cooling of nuclear reactors etc. Taking into account its importance, Raju et al. (2015) made a theoretical analysis to study the effect of induced magnetic field on free convection flow of viscous dissipative fluid past a vertical plate. Further, investigation of unsteady MHD flow under different configurations and conditions finds application in many natural and engineering processes because fluid transients may be expected at the start-up time of so many natural phenomena, industrial processes and devices, namely, MHD power generators, MHD pumps, MHD accelerators, electronic equipment and devices, thermonuclear reactors, casting and levitation, atmospheric winds etc. Keeping in view importance of such study, several researchers investigated hydromagnetic natural convection flow with heat and mass transfer through a fluid saturated porous medium near vertical plate considering different aspects of the problem. Relevant studies are due to Hossain and Mandal (1985), Jha (1991), Ibrahim et al. (2004), Chaudhary and Jain (2007), Makinde and Sibanda (2008), Eldabe et al. (2011) and Umamaheswar et al. (2015a). Umamaheswar et al. (2015b) investigated an unsteady MHD free convection flow of a double diffusive viscoelastic fluid past an inclined permeable plate in the presence of viscous dissipation and heat absorption.

Space laboratories were established on the concept of gray body radiation of its optical measurement. Radiation from the external sources, which passes through the media from its boundary to a given point, diminishes gradually at the expense of absorption. The principle of radiation is based on comparing the quality of heat radiated by the solid to another solid whose radiation factor or emissivity is known. The emissivity of gray body depends on its nature, temperature, state of emitting surface and is generally determined by the experiments. Thermal radiation effect plays a vital role in a numerous applications, viz. condensed fuel combustion (Chen et al., 1993), solar energy collectors (Reddy and Kumar, 2008), heat exchangers (Nassab and Maramisaram, 2009), glass and ceramic production (Obidina and Kiseleva, 1980), rocket propulsion (Saladino and Farmer, 1993), laser processing of materials (Gedda et al., 2002) etc. It may be noted that energy equation in the presence of radiation becomes non-linear. Due to this reason some reasonable approximations, which are proposed to

${ }^{*}$ Corresponding author. Email: seth.gs.am@ismdhanbad.ac.in 
linearize the energy equation, are Schuster-Schwartzchild two flux model, Milne-Eddington approximation, Cogley approximation (Cogley et al., 1968) and Rosseland approximation (Siegel and Howell, 1972).

Takhar et al. (1996) analysed the effects of radiation on hydromagnetic free convection flow of a radiating gas past a semi-infinite vertical plate. Raptis and Massalas (1998) investigated two dimensional unsteady hydromagnetic flow with heat transfer of an optically thick radiating fluid in the presence of thermal radiation and viscous and joule dissipations using Rosseland approximation whereas Raptis et al. (2004) considered hydromagnetic asymmetric flow of an electrically conducting and optically thick radiating fluid past a semi-infinite stationary plate considering Rosseland approximation. Azzam (2002) presented an analysis of the effect of thermal radiation on MHD mixed convection flow near a semi-infinite moving vertical plate when the temperature differences are very high. Aboeldahab and Azzam (2005) discussed hydromagnetic mixed convection flow with radiative heat transfer of a gray, optically thick radiating, electrically conducting and viscous fluid past a semi-infinite inclined plate using Rosseland approximation. Mbeledogu and Ogulu (2007) studied unsteady hydromagnetic natural convection heat and mass transfer flow of a radiating fluid past a vertical porous plate in the presence of thermal radiation. Palani and Abbas (2009) obtained numerical solution for hydromagnetic free convection flow past an impulsively started isothermal vertical plate in the presence of thermal radiation. Prakash et al. (2013) analysed the effect of thermal radiation on unsteady hydromagnetic flow past an impulsively started infinite vertical plate through porous medium with variable temperature taking Dufour effects into account. Hussanan et al. (2014) investigated unsteady MHD natural convection flow of a radiating fluid over an oscillating vertical plate with constant mass diffusion and Newtonian heating. Ghosh et al. (2015) analysed transient hydromagnetic natural convection flow with heat and mass transfer of an optically thick radiating fluid past an impulsively moving vertical plate in the presence of thermal radiation and mass diffusion by considering Rosseland approximation.

The growing requirement for chemical reactions in chemical and metallurgical industries necessitates the study of heat and mass transfer with chemical reaction through porous medium which are of considerable importance in many processes. There are so many transport processes which are governed by the combined action of thermal and solutal buoyancy forces in the presence of chemical reaction. These processes occur in nuclear reactor safety and combustion systems, solar collectors as well as chemical and metallurgical engineering. In addition to it, other application include solidification of binary alloys and crystal growth, dispersion of dissolved materials or particulate water in flows, drying and dehydration operation in chemical and food processing plants and combustion of atomized liquid fuels. There are two types of reactions, homogeneous reaction and heterogeneous reaction. A homogeneous reaction is one that occurs uniformly throughout a given phase. The species generation in a homogeneous reaction is analogous to internal source of heat generation. In contrast, a heterogeneous reaction takes place in a restricted region or within the boundary of a phase. It can, therefore, be treated as a boundary condition similar to the constant heat flux condition in heat transfer. Fairbanks and Wike (1950) initiated the study of diffusion and chemical reaction in an isothermal laminar flow along a soluble flat plate. Chambre and Young (1958) investigated the diffusion of a chemically reactive species in a laminar boundary layer flow over a flat plate. Modather et al. (2009) made an analytical study of the effects of permeability and chemical reaction on heat and mass transfer near an infinite moving vertical permeable plate in a fluid saturated porous medium. Rashad et al. (2011) analysed hydromagnetic free convection heat and mass transfer of a chemically reactive fluid from radiative stretching surface embedded in a fluid saturated porous medium. Chamkha et al. (2012) considered heat and mass transfer from truncated cones with variable wall temperature and concentration in the presence of chemical reaction. Muthucumaraswamy et al. (2013) discussed the effect of rotation and chemical reaction of first order on unsteady hydromagnetic free convection flow of a fluid past a uniformly accelerated isothermal vertical plate with variable mass diffusion. Ahmed and Kalita (2013) made analytical and numerical study of hydromagnetic natural convection flow due to heat and mass transfer of an electrically conducting optically thin radiating and chemically reactive fluid near an infinite vertical oscillating plate embedded in a fluid saturated porous medium with variable temperature and variable concentration at the surface of the plate. Seth et al. (2014) considered unsteady hydromagnetic natural convection flow with heat and mass transfer past an impulsively moving vertical plate in a rotating system in the presence of thermal radiation, chemical reaction and Hall effects. ElKabeir et al. (2015) investigated the effects of thermal and solutal buoyancy forces on unsteady three dimensional natural convection boundary layer flow of a chemically reactive fluid near a moving vertical plate embedded in a fluid saturated porous medium with Soret and Dufour effects.

In all the above studies, the applied magnetic field is assumed to be acting perpendicular to the direction of fluid flow. However, in the problems of practical interest, viz. MHD power generation, magnetic material processing flow control and in other geophysical problems, the magnetic field may act obliquely to the fluid flow. Thus, it is worthwhile to consider the case when the applied magnetic field acts in a direction which is inclined at an angle $\alpha$ (less than $\pi / 2$ ) to the vertical direction of the fluid flow. Ghosh and Bhattacharjee (2000) and Seth et al. (2010) investigated steady Hartmann flow within a parallel plate rotating channel in the presence of inclined magnetic field with Hall effects considering different aspects of the problem. Seth et al. (2009) discussed the effects of Hall current on unsteady hydromagnetic Couette flow in a rotating system in the presence of inclined magnetic field. Beg et al. (2011) considered oscillatory Couette flow of a viscous, incompressible and electrically conducting fluid within a parallel plate rotating channel in a highly permeable fluid-saturated porous medium under the influence of an oblique magnetic field. Akram et al. (2014) made an analytical study of peristaltic flow with heat and mass transfer of a Bingham fluid within a channel with different wave forms in the presence of inclined magnetic field. Contribution on this topic is also due to Reddy et al. (2008).

In the above mentioned studies, analytical/numerical solution is obtained under different thermal conditions at the plate which are continuous and well defined. However, there exist several practical problems which involve non-uniform or arbitrary thermal conditions. Some of the industry based applications include nuclear heat transfer control, materials processing, turbine blade heat transfer, electronic circuits and sealed gas-filled enclosure heat transfer operations. Several researchers, namely, Hayday et al. (1967), Kelleher (1971), Kao (1975) and Lee and Yovanovich (1991) analysed natural convection flow from a vertical plate with discontinuous thermal boundary conditions considering different aspects of the problem. Chandran et al. (1998) studied unsteady natural convective flow of a viscous and incompressible fluid near a vertical plate with ramped temperature. Patra et al. (2012) analyzed transient approach to radiative heat transfer free convection flow with ramped wall temperature. Seth et al. (2013) discussed unsteady hydromagnetic natural convection flow past an impulsively moving vertical plate with ramped temperature through a fluid saturated porous medium in a rotating system in the presence of thermal radiation. Nandkeolyar and Das (2015) investigated the effects of thermal radiation on unsteady hydromagnetic natural convection flow of an optically thick radiating fluid past an impulsively moving vertical plate with ramped temperature embedded in a fluid saturated porous medium in the presence of inclined magnetic field. Khalid et al. (2015) made analytical study of unsteady free convection flow of nanofluid near an oscillating infinite vertical plate with ramped wall temperature.

Authors are motivated to analyze unsteady hydromagnetic free convection flow with heat and mass transfer of an optically thick radiating fluid near impulsively moving vertical plate with ramped heat flux embedded in a fluid saturated porous medium in the presence of 
inclined magnetic field and first order chemical reaction. As per authors' knowledge, this problem has not yet been investigated. However, it may have bearings on the problems of practical interest, namely, nuclear heat transfer control, electronic circuits, sealed gas-field enclosure heat transfer operations, fabrication of thin film photovoltaic devices, fire dynamics insulations, recovery of petroleum products and gases (e.g. CBM: Coal Bed Methane and UCG: Underground Coal Gasification) etc.

\section{FORMULATION OF THE PROBLEM}

Consider unsteady laminar free convection flow with heat and mass transfer of an electrically-conducting, viscous, incompressible, optically thick radiating and chemically-reactive fluid through a uniform porous medium bounded by an impulsively moving infinite vertical plate with ramped heat flux in the presence of uniform inclined magnetic field. Cartesian co-ordinate system is chosen in such a way that $x$-axis is taken along the plate in a vertically upward direction and $y$-axis is normal to the plane of the plate in the fluid. The fluid is permeated by uniform magnetic field $B_{0}$, which is applied in a direction that makes an angle $\alpha$ with $x$-axis in $x y$ - plane. Initially, i.e. at time $t^{\prime} \leq 0$, both the fluid and plate are at rest and are maintained at a uniform temperature $T_{\infty}$ and species concentration $C_{\infty}$. At time $t^{\prime}>0$, plate starts moving in $x$ - direction with uniform velocity $U_{0}$ in its own plane. The species concentration at the surface of the plate is raised to $C_{w}$ and the heat supplied from the plate to the fluid is raised at the rate of $q \cdot \frac{t^{\prime}}{t_{0}}$ when $0<t^{\prime} \leq t_{0}$ and $q$ when $t^{\prime}>t_{0}$ ( $q$ and $t_{0}$ being the constant heat flux and characteristic time respectively). It is assumed that there exists a homogeneous chemical reaction of first order with constant rate $k_{2}$ between the diffusing species and the fluid. Schematic diagram of the physical problem is presented in Fig. 1.

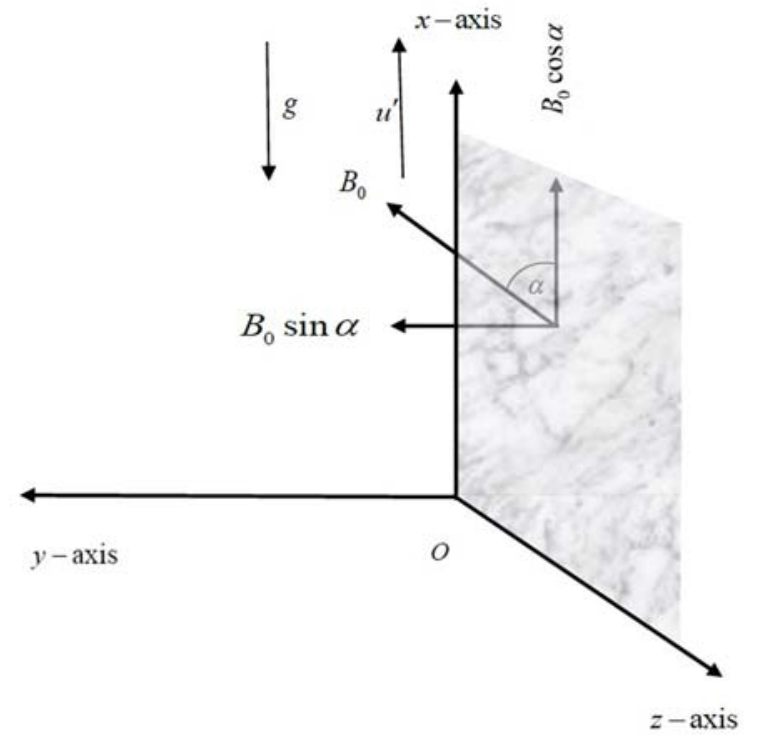

Fig. 1 Schematic diagram of the physical problem

Since the plate is of infinite extent in $x$ and $z$ - directions and is electrically non-conducting, all physical quantities are functions of $y$ and $t^{\prime}$ only. The fluid under investigation is an electrically conducting metallic liquid or partially ionized fluid. Due to this reason the induced magnetic field produced by the fluid motion is negligible so that the magnetic field $\vec{B} \equiv\left(B_{0} \cos \alpha, B_{0} \sin \alpha, 0\right)$. In addition to it, no external electric field is applied, therefore, the effect of polarization of fluid is negligible. Thus $\vec{E} \equiv(0,0,0)$.

Under usual Boussinesq's approximation, the governing equations for hydromagnetic free convection flow of a radiating and chemically reactive fluid are

$$
\begin{aligned}
& \frac{\partial u^{\prime}}{\partial t^{\prime}}=v \frac{\partial^{2} u^{\prime}}{\partial y^{2}}+g \beta\left(T-T_{\infty}\right)+g \beta^{*}\left(C-C_{\infty}\right)-\frac{v}{k_{1}} u^{\prime}-\frac{\sigma B_{0}^{2}}{\rho} \sin ^{2} \alpha u^{\prime}, \\
& \rho C_{p} \frac{\partial T}{\partial t^{\prime}}=k \frac{\partial^{2} T}{\partial y^{2}}-\frac{\partial q_{r}}{\partial y}, \\
& \frac{\partial C}{\partial t^{\prime}}=D_{m} \frac{\partial^{2} C}{\partial y^{2}}-k_{2}\left(C-C_{\infty}\right),
\end{aligned}
$$

where, $u^{\prime}, T, C, D_{m}, g, v, \rho, k, \beta, \beta^{*}, k_{1}, \sigma, C_{p}, q_{r}$ and $k_{2}$ are fluid velocity, fluid temperature, species concentration, mass diffusivity, acceleration due to gravity, kinematic coefficient of viscosity, fluid density, thermal conductivity, thermal expansion coefficient, concentration expansion coefficient, permeability of the medium, electrical conductivity, specific heat at constant pressure, radiative heat flux and chemical reaction coefficient respectively.

The initial and boundary conditions are:

$t^{\prime} \leq 0: u^{\prime}=0, T=T_{\infty}, C=C_{\infty}$ for all $y \geq 0$

$$
\begin{aligned}
& t^{\prime}>0: u^{\prime}=U_{0}, C=C_{w}, \\
& \frac{\partial T}{\partial y}= \begin{cases}-\frac{q}{k} \cdot \frac{t^{\prime}}{t_{0}} \text { for } 0<t^{\prime} \leq t_{0} & \text { at } y=0 \\
-\frac{q}{k} & \text { for } t^{\prime}>t_{0}\end{cases} \\
& t^{\prime}>0: u^{\prime} \rightarrow 0, T \rightarrow T_{\infty}, C \rightarrow C_{\infty} \text { as } y \rightarrow \infty .
\end{aligned}
$$

For an optically thick radiating fluid the radiative heat flux can obtained with the help of Rosseland approximation (Siegel and Howell, 1972). Its expression is derived using diffusion concept of radiative heat transfer which is given by

$q_{r}=-\frac{4 \sigma^{*}}{3 k^{*}} \frac{\partial T^{4}}{\partial y}$,

where $\sigma^{*}$ is the Stefan-Boltzman constant and $k^{*}$ is the Rosseland mean absorption coefficient of the medium. We assume that the temperature difference between the fluid temperature $T$ in the boundary layer region and fluid temperature $T_{\infty}$ in the free stream is sufficiently small so that equation (5) can be linearized by expanding $T^{4}$ in Taylor series about free stream temperature $T_{\infty}$ which, after neglecting second and higher order terms, takes the form

$T^{4} \cong 4 T_{\infty}^{3} T-3 T_{\infty}^{4}$.

In view of equations (5) and (6), equation (2) reduces to

$\rho C_{p} \frac{\partial T}{\partial t^{\prime}}=k\left(1+\frac{16 \sigma^{*} T_{\infty}^{3}}{3 k k^{*}}\right) \frac{\partial^{2} T}{\partial y^{2}}$.

To convert equations (1), (3) and (7) in non-dimensional form, we introduce the following non-dimensional quantities: 


$$
\left.\begin{array}{l}
\eta=\frac{y U_{0}}{v}, t=\frac{t^{\prime}}{t_{0}}, t_{0}=\frac{v}{U_{0}^{2}}, u=\frac{u^{\prime}}{U_{0}}, \\
\theta=\frac{k\left(T-T_{\infty}\right) U_{0}^{2}}{q v}, \phi=\frac{C-C_{\infty}}{C_{w}-C_{\infty}} .
\end{array}\right\}
$$

The equations (1), (3) and (7), with the help of (8), are converted in non-dimensional form which are given by

$\frac{\partial u}{\partial t}=\frac{\partial^{2} u}{\partial \eta^{2}}+G_{r} \theta+G_{c} \phi-\left(M \sin ^{2} \alpha+\frac{1}{K_{1}}\right) u$,

$\delta \frac{\partial \theta}{\partial t}=\frac{\partial^{2} \theta}{\partial \eta^{2}}$

$\frac{\partial \phi}{\partial t}=\frac{1}{S_{c}} \frac{\partial^{2} \phi}{\partial \eta^{2}}-\gamma \phi$

where, $\delta=\frac{P_{r}}{(1+R)}$ is the effective Prandtl number, $R=\frac{16 \sigma^{*} T_{\infty}^{3}}{3 k k^{*}}$ is the radiation parameter, $P_{r}=\frac{\rho v C_{p}}{k}$ is the Prandtl number, $G_{r}=\frac{g \beta q v^{2}}{k U_{0}^{4}}$ is the thermal Grashof number, $G_{c}=\frac{g \beta^{*} v\left(C_{w}-C_{\infty}\right)}{U_{0}^{3}}$ is the solutal Grashof number, $\gamma=\frac{k_{2} v}{U_{0}^{2}}$ is the chemical reaction parameter, $S_{c}=\frac{v}{D_{m}}$ is the Schmidt number, $M=\frac{\sigma B_{0}^{2} v}{\rho U_{0}^{2}}$ is the Magnetic parameter and $K_{1}=\frac{k_{1} U_{0}^{2}}{v^{2}}$ is the permeability parameter.

The initial and boundary conditions (4a) to (4c), in non-dimensional form, are presented as

$t \leq 0: u=0, \theta=0, \phi=0$ for $\eta \geq 0$,

$t>0: u=1, \phi=1$,

$\frac{\partial \theta}{\partial \eta}=\left\{\begin{array}{l}-t \text { for } 0<t \leq 1 \\ -1 \text { for } t>1\end{array} \quad\right.$ at $\eta=0$

$t>0: u \rightarrow 0, \theta \rightarrow 0, \phi \rightarrow 0$ as $\eta \rightarrow \infty$

\section{SOLUTION OF THE PROBLEM}

In order to obtain the exact solution of the present problem we have used Laplace transform technique. Taking Laplace transform of equations (9) to (11) and using initial conditions (12a), we obtain

$$
\begin{aligned}
& \frac{d^{2} \bar{u}}{d \eta^{2}}-(s+a) \bar{u}+G_{r} \bar{\theta}+G_{c} \bar{\phi}=0, \\
& \frac{d^{2} \bar{\theta}}{d \eta^{2}}-s \delta \bar{\theta}=0, \\
& \frac{d^{2} \bar{\phi}}{d \eta^{2}}-S_{c}(s+\gamma) \bar{\phi}=0,
\end{aligned}
$$

where

$$
\left.\begin{array}{l}
\bar{u}(\eta, s)=\int_{0}^{\infty} u(\eta, t) e^{-s t} d t, \bar{\theta}(\eta, s)=\int_{0}^{\infty} \theta(\eta, t) e^{-s t} d t, \\
\bar{\phi}(\eta, s)=\int_{0}^{\infty} \phi(\eta, t) e^{-s t} d t \text { and } a=\left(M \sin ^{2} \alpha+\frac{1}{K_{1}}\right) .
\end{array}\right\}
$$

The boundary conditions (12b) and (12c), with the use of Laplace transform, reduce to

$$
\begin{aligned}
& \bar{u}=\frac{1}{s}, \bar{\phi}=\frac{1}{s}, \frac{d \bar{\theta}}{d \eta}=-\frac{1}{s^{2}}\left(1-e^{-s}\right) \text { at } \eta=0, \\
& \bar{u} \rightarrow 0, \bar{\phi} \rightarrow 0, \bar{\theta} \rightarrow 0 \text { as } \eta \rightarrow \infty .
\end{aligned}
$$

Solution of equations (13) to (15) subject to the boundary conditions $(17 \mathrm{a})$ and $(17 \mathrm{~b})$ is given by

$$
\begin{aligned}
\bar{u}(\eta, s) & =\frac{1}{s} e^{-\eta \sqrt{a+s}}+\frac{G_{r}}{\sqrt{\delta}} \frac{\left(1-e^{-s}\right)}{\delta^{\prime}\left(s-a_{1}\right) s^{\frac{5}{2}}}\left\{e^{-\eta \sqrt{a+s}}-e^{-\eta \sqrt{\delta s}}\right\} \\
& +\frac{G_{c}}{S_{c}{ }^{\prime}\left(s-b_{1}\right) s}\left\{e^{-\eta \sqrt{a+s}}-e^{-\eta \sqrt{s_{c}(s+\gamma)}}\right\}, \\
\bar{\theta}(\eta, s) & =\frac{1}{\sqrt{\delta}} \frac{\left(1-e^{-s}\right)}{s^{\frac{5}{2}}} e^{-\eta \sqrt{\delta s}}, \\
\bar{\phi}(\eta, s) & =\frac{1}{s} e^{-\eta \sqrt{s_{c}(s+\gamma)}}
\end{aligned}
$$

where, $a_{1}=\frac{a}{\delta^{\prime}}, b_{1}=\frac{b}{S_{c}{ }^{\prime}}, \delta^{\prime}=\delta-1, S_{c}{ }^{\prime}=S_{c}-1$ and $b=a-S_{c} \gamma$.

The solution for fluid velocity $u(\eta, t)$, fluid temperature $\theta(\eta, t)$ and species concentration $\phi(\eta, t)$ is obtained by taking inverse Laplace transform of equations (18) to (20) and is presented in the following form

$$
\begin{aligned}
u(\eta, t)= & f_{1}(\eta, a, 0,1, t)+\frac{G_{r}}{\delta^{\prime} \sqrt{\delta}}\left\{f_{2}\left(\eta, a, a_{1}, \delta, t\right)\right. \\
& \left.-H(t-1) f_{2}\left(\eta, a, a_{1}, \delta, t-1\right)\right\}+\frac{G_{c}}{b} \times \\
& {\left[\left\{f_{1}\left(\eta, a, b_{1}, 1, t\right)-f_{1}(\eta, a, 0,1, t)\right\}\right.} \\
& \left.-\left\{f_{1}\left(\eta, \gamma, b_{1}, S_{c}, t\right)-f_{1}\left(\eta, \gamma, 0, S_{c}, t\right)\right\}\right], \\
\theta(\eta, t)= & \frac{1}{\sqrt{\delta}}\left[f_{3}(\eta \sqrt{\delta}, t)-H(t-1) f_{3}(\eta \sqrt{\delta}, t-1)\right], \\
\phi(\eta, t)= & \frac{1}{2}\left[e^{\eta \sqrt{s_{c} \gamma}} \operatorname{erfc}\left(\sqrt{\gamma t}+\frac{\eta \sqrt{S_{c}}}{2 \sqrt{t}}\right)+e^{-\eta \sqrt{S_{c} \gamma}} \times\right. \\
& \left.\operatorname{erfc}\left(-\sqrt{\gamma t}+\frac{\eta \sqrt{S_{c}}}{2 \sqrt{t}}\right)\right],
\end{aligned}
$$

where,

$$
\begin{aligned}
f_{1}\left(\eta, \xi_{1}, \xi_{2}, \xi_{3}, t\right) & =\frac{e^{\xi_{2} t}}{2}\left[e^{\eta \sqrt{\left(\xi_{1}+\xi_{2}\right) \xi_{3}}} \operatorname{erfc}\left(\sqrt{\left(\xi_{1}+\xi_{2}\right) t}+\frac{\eta \sqrt{\xi_{3}}}{2 \sqrt{t}}\right)\right. \\
+ & \left.e^{-\eta \sqrt{\left(\xi_{1}+\xi_{2}\right) \xi_{3}}} \operatorname{erfc}\left(-\sqrt{\left(\xi_{1}+\xi_{2}\right) t}+\frac{\eta \sqrt{\xi_{3}}}{2 \sqrt{t}}\right)\right], \\
f_{2}\left(\eta, \xi_{4}, \xi_{5}, \xi_{6}, t\right) & =\int_{0}^{t}\left\{f_{2}^{\prime}\left(\eta, \xi_{4}, \xi_{5}, 1, v\right)-f_{2}^{\prime}\left(\eta, 0, \xi_{5}, \xi_{6}, v\right)\right\} \times \\
& \frac{4}{3 \sqrt{\pi}}(t-v)^{\frac{3}{2}} d v,
\end{aligned}
$$




$$
\begin{aligned}
& f_{2}^{\prime}\left(\eta, \xi_{7}, \xi_{8}, \xi_{9}, v\right)=\frac{e^{\xi_{8} v}}{2}\left[e^{\eta \sqrt{\left(\xi_{7}+\xi_{8}\right) \xi_{9}}} \operatorname{erfC}\left(\sqrt{\left(\xi_{7}+\xi_{8}\right) v}+\frac{\eta \sqrt{\xi_{9}}}{2 \sqrt{v}}\right)\right. \\
& \left.+e^{-\eta \sqrt{\left(\xi_{7}+\xi_{8}\right) \xi_{9}}} \operatorname{erfc}\left(-\sqrt{\left(\xi_{7}+\xi_{8}\right) v}+\frac{\eta \sqrt{\xi_{9}}}{2 \sqrt{v}}\right)\right] \\
& f_{3}\left(\xi_{10}, t\right)=\frac{1}{3} \sqrt{\frac{t}{\pi}}\left(4 t+\xi_{10}{ }^{2}\right) e^{-\frac{\xi_{10}{ }^{2}}{4 t}}-\frac{\xi_{10}}{6}\left(6 t+\xi_{10}{ }^{2}\right) \operatorname{erfc}\left(\frac{\xi_{10}}{2 \sqrt{t}}\right) \text {. }
\end{aligned}
$$

It may be noted that the solution (21) is valid when $\delta \neq 1$ and $S_{c} \neq 1$. Moreover the solutions of fluid velocity for other three cases of interest are presented below:

Case I: When $\delta \neq 1$ and $S_{c}=1$,

$$
\begin{aligned}
u(\eta, t)= & f_{1}(\eta, a, 0,1, t)+\frac{G_{r}}{\delta^{\prime} \sqrt{\delta}}\left\{f_{2}\left(\eta, a, a_{1}, \delta, t\right)-H(t-1) \times\right. \\
& \left.f_{2}\left(\eta, a, a_{1}, \delta, t-1\right)\right\}+\frac{G_{c}}{(\gamma-a)}\left\{f_{4}(\eta, a, t)-f_{4}(\eta, \gamma, t)\right\},
\end{aligned}
$$

where,

$$
f_{4}\left(\eta, \xi_{11}, t\right)=\frac{1}{2}\left[e^{\eta \sqrt{\xi_{11}}} \operatorname{erfc}\left(\sqrt{\xi_{11} t}+\frac{\eta}{2 \sqrt{t}}\right)+e^{-\eta \sqrt{\xi_{11}}} \operatorname{erfc}\left(-\sqrt{\xi_{11} t}+\frac{\eta}{2 \sqrt{t}}\right)\right] \text {. }
$$

Case II: When $\delta=1$ and $S_{c} \neq 1$,

$$
\begin{aligned}
u(\eta, t)= & f_{1}(\eta, a, 0,1, t)-\frac{G_{r}}{a}\left\{f_{5}(\eta, t)-H(t-1) f_{5}(\eta, t-1)\right\} \\
& +\frac{G_{c}}{b}\left[\left\{f_{1}\left(\eta, a, b_{1}, 1, t\right)-f_{1}(\eta, a, 0,1, t)\right\}\right. \\
& \left.-\left\{f_{1}\left(\eta, \gamma, b_{1}, S_{c}, t\right)-f_{1}\left(\eta, \gamma, 0, S_{c}, t\right)\right\}\right]
\end{aligned}
$$

where,

$$
\begin{aligned}
f_{5}(\eta, t) & =\int_{0}^{t} \frac{2 \eta}{3 \pi} \frac{e^{-\left(a v+\frac{\eta^{2}}{4 v}\right)}(t-v)^{\frac{3}{2}} d v-\left\{\frac{\sqrt{t}}{3 \sqrt{\pi}}\left(4 t+\eta^{2}\right) e^{-\frac{\eta^{2}}{4 t}}\right.}{v^{\frac{3}{2}}} \\
- & \left.\frac{\sqrt{\delta}}{6}\left(6 t+\eta^{2}\right) \operatorname{erfc}\left(\frac{\sqrt{\delta}}{2 \sqrt{t}}\right)\right\} .
\end{aligned}
$$

Case III: When $\delta=1$ and $S_{c}=1$,

$$
\begin{aligned}
u(\eta, t) & =f_{1}(\eta, a, 0,1, t)-\frac{G_{r}}{a}\left\{f_{5}(\eta, t)-H(t-1) f_{5}(\eta, t-1)\right\} \\
& +\frac{G_{c}}{(\gamma-a)}\left\{f_{4}(\eta, a, t)-f_{4}(\eta, \gamma, t)\right\} .
\end{aligned}
$$

Here, $\xi_{i}(i=1, \ldots, 11), f_{i}(i=1, \ldots, 5)$ and $H(t-1)$ are, respectively, dummy variables, dummy functions and Heaviside unit step function.

\subsection{SKIN FRICTION, SHERWOOD NUMBER AND PLATE TEMPERATURE}

The expressions for the skin friction $\tau$ and Sherwood number $S_{h}$ which are measures of Shear stress at the plate and rate of mass transfer at the plate are given by

$$
\begin{aligned}
\tau & =-\left.\frac{\partial u}{\partial \eta}\right|_{\eta=0} \\
& =\frac{1}{\sqrt{\pi t}} e^{-a t}+\sqrt{a} \operatorname{erf}(\sqrt{a t})+\frac{G_{r}}{\delta^{\prime} \sqrt{\delta}} \times
\end{aligned}
$$

$$
[G(0, t)-H(t-1) G(0, t-1)]+\frac{G_{c}}{b} \times P(0, t),
$$

where,

$$
\begin{aligned}
G(0, t)= & \int_{0}^{t} \frac{4}{3 \sqrt{\pi}}(t-v)^{\frac{3}{2}} e^{a_{1} v}\left\{\frac{e^{-a_{1} v}}{\sqrt{\pi v}}\left(e^{-a v}-\sqrt{\delta}\right)+\sqrt{a+a_{1}} \times\right. \\
& \left.\operatorname{erf}\left(\sqrt{\left(a+a_{1}\right) v}\right)+\sqrt{a_{1} \delta} \operatorname{erf}\left(\sqrt{a_{1} v}\right)\right\} d v, \\
P(0, t)= & e^{b_{1} t}\left\{\left(\sqrt{\frac{1}{\pi t}} e^{-\left(a+b_{1}\right) t}+\sqrt{\left(a+b_{1}\right)} \operatorname{erf}\left(\sqrt{\left(a+b_{1}\right) t}\right)\right)\right. \\
& \left.-\left(\sqrt{\frac{S_{c}}{\pi t}} e^{-\left(\gamma+b_{1}\right) t}+\sqrt{S_{c}\left(\gamma+b_{1}\right)} \operatorname{erf}\left(\sqrt{\left(\gamma+b_{1}\right) t}\right)\right)\right\} \\
& -\left\{\left(\sqrt{\frac{1}{\pi t}} e^{-a t}+\sqrt{a} \operatorname{erf}(\sqrt{a t})\right)-\left(\sqrt{\frac{S_{c}}{\pi t}} e^{-\gamma t}+\sqrt{S_{c} \gamma} \operatorname{erf}(\sqrt{\gamma t})\right)\right\} . \\
S_{h}=- & \left.\frac{\partial \phi}{\partial \eta}\right|_{\eta=0} \\
= & \sqrt{\frac{S_{c}}{\pi t}} e^{-\gamma t}+\sqrt{S_{c} \gamma} \operatorname{erf}(\sqrt{\gamma t}) .
\end{aligned}
$$

The temperature at the plate, in non-dimensional form, is given by

$$
\theta(0, t)=\frac{4}{3 \sqrt{\pi \delta}}\left[t^{\frac{3}{2}}-H(t-1)(t-1)^{\frac{3}{2}}\right]
$$

\section{RESULTS AND DISCUSSION}

In order to analyze the effects of magnetic field, thermal radiation, thermal buoyancy force, solutal buoyancy force, chemical reaction, angle of inclination of magnetic field and time on the flow-field, numerical values of fluid velocity $u(\eta, t)$ in the boundary layer region, computed from the solution (21), are displayed graphically versus boundary layer co-ordinate $\eta$ in Figs. 2 to 8 for various values of magnetic parameter $M$, effective Prandtl number $\delta$, thermal Grashof number $G_{r}$, solutal Grashof number $G_{c}$, chemical reaction parameter $\gamma$, angle of inclination of magnetic field $\alpha$ and time $t$ taking Prandtl number $P_{r}=0.71$, Permeability parameter $K_{1}=0.2$ and Schmidt number $S_{c}=0.6$.

Figure 2 depicts the influence of magnetic field on the fluid velocity. It is perceived from Fig. 2 that, fluid velocity $u$ decreases on increasing $M$. This implies that magnetic field tends to retard fluid velocity in the boundary layer region. This is due to the fact that the application of a magnetic field always results in a resistive-type force (Lorentz force) which is similar to the drag force and upon increasing the values of $M$ the drag force increases and tends to resist the fluid flow and thus reduces the fluid motion significantly. Figure 3 illustrates the influence of effective Prandtl number on the fluid velocity. It is noticed from this Fig. that there is steady decrease in the fluid velocity with the increase in $\delta$. Since $\delta=\frac{P_{r}}{(1+R)}, \delta$ decreases on increasing $R$ for fixed value of $P_{r}$.

That is there is a continuous increase in fluid velocity on increasing the values of $R$. Thus we conclude that, for an optically thick radiating fluid, thermal radiation tends to accelerate fluid velocity in the boundary layer region. This is justified because thermal radiation tends to enhance fluid temperature within the boundary layer region (Fig. 9). As a consequence 
the thickness of both momentum and thermal boundary layers increases on increasing $R$ and heat transfer rate reduces in the presence of thermal radiation. The effects of thermal and solutal buoyancy forces on the velocity field are shown in Figs. 4 and 5. It is apparent from Figs. 4 and 5 that the effects of $G_{r}$ and $G_{c}$ on fluid velocity are similar. Fluid velocity $u$ increases on increasing $G_{r}$ and $G_{c}$. Thus thermal and solutal buoyancy forces have tendency to accelerate fluid velocity in the boundary layer region. Physically, it is possible because an increase in the value of $G_{r}$ and $G_{c}$ has a tendency to increase the thermal and solutal buoyancy effects. The thermal Grashof number signifies the relative importance of thermal buoyancy force to the viscous force whereas the solutal Grashof number defines the ratio of the solutal buoyancy force to the viscous force. Increase in thermal and solutal Grashof numbers indicates smaller viscous effect than thermal and solutal buoyancy effects in the momentum equation and consequently, causes increase in the fluid velocity. In addition, it is seen that peak values of the velocity increases rapidly near the plate as thermal and solutal Grashof numbers increase and then decays smoothly to the free stream velocity. The change in velocity profile due to chemical reaction is displayed in Fig. 6. This figure indicates the fact that the fluid motion is retarded on account of chemical reaction in the boundary layer region. This elucidates that the consumption of chemical species leads to fall in the concentration field (Fig. 12) which in turn diminishes the buoyancy effects due to concentration gradients. Consequently, the fluid flow is decelerated. Figure 7 depicts the effect of angle of inclination of magnetic field on the fluid velocity. It is evident from Fig. 7 that $u$ decreases on increasing $\alpha$. This implies that the strength of resistive force, known as Lorentz force which is induced due to presence of magnetic field in the flow-field, increases on increasing angle of inclination of magnetic field $\alpha$ and its strength becomes maximum when $\alpha=\pi / 2$, i. e. when applied magnetic field is in the transverse direction to the fluid flow. It is well known that the tendency of Lorentz force is to supress the fluid motion. If it is required to accelerate fluid flow in practical problems of interest, then in place of applying magnetic field in the vertical direction, it may be applied in another direction which is inclined with the direction of the flow. By changing the angle of inclination, fluid velocity may be accelerated /retarded. Figure 8 exhibits the influence of time on the fluid velocity. It is revealed from Fig. 8 that $u$ increases on increasing $t$. This implies that velocity field is getting enhanced with the progress of time in the boundary layer region.

In order to study the influence of thermal radiation and time on the temperature field, numerical values of fluid temperature $\theta(\eta, t)$ in the boundary layer region, computed from the solution (22), are presented graphically versus boundary layer co-ordinate $\eta$ in Figures 9 and 10 for various values of $\delta$ and $t$ taking $P_{r}=0.71$.

Figure 9 depicts the influence of effective Prandtl number on the fluid temperature. It is revealed from Fig. 9 that $\theta$ decreases on increasing $\delta$. This implies that thermal radiation tends to enhance the fluid temperature in the boundary layer region. Figure 10 exhibits the influence of time on the fluid temperature. It is found from Fig. 10 that the effect of time $t$ on the fluid temperature is similar to that of velocity profiles. Finally, it is observed from the temperature profiles that the fluid temperature is maximum near the plate and it decreases away from the plate and finally asymptotically approaches to zero in the free stream region.

To study the effects of mass diffusion, chemical reaction and time on species concentration, the numerical values of species concentration $\phi(\eta, t)$ in the boundary layer region, computed from the solution (23), are presented graphically versus boundary layer co-ordinate $\eta$ in Figs. 11 to 13 for various values of $S_{c}, \gamma$ and $t$.
It is evident from Fig. 11 that $\phi$ decreases on increasing $S_{c}$, i. e. concentration level of the fluid drops due to increasing Schmidt number indicating the fact that the mass diffusivity raises the concentration level steadily in the boundary layer region. In order to concentrate more on the numerical values of the results obtained in this study, we have chosen the values of $S_{c}$ in such a way that they represent the diffusion chemical species of common interest in air, for example, $S_{c}=0.22,0.6,0.78$ and 2.62. Physically, they are representing diffusing chemical species of Hydrogen, water vapour in air, Ammonia and Propyl Benzene respectively at $25^{\circ} \mathrm{C}$ temperature and 1 atmospheric pressure. It is clearly seen that concentration level of hydrogen is more than that of water vapor. Further it is observed from Figs. 12 and 13 that that $\phi$ decreases on increasing $\gamma$ whereas it increases on increasing $t$. This implies that chemical reaction tends to reduce species concentration whereas time has a reverse effect on it within the boundary layer region. Also, it is noticed from all the concentration profiles that species concentration attains its maximum value near the surface of the plate and then decreases properly on increasing boundary layer coordinate $\eta$ to approach the free stream value.

Numerical values of skin friction $\tau$, computed from (27), are presented in tabular form in Tables 1 and 2 for various values of $M, \delta$, $G_{r}, G_{c}, \gamma, \alpha$ and $t$ taking $P_{r}=0.71, K_{1}=0.2$ and $S_{c}=0.6$. It is clear from Tables 1 and 2 that $\tau$ increases on increasing $M, \delta, G_{r}, \gamma, t$ and $\alpha$ whereas it decreases on increasing $G_{c}$. This implies that magnetic field, thermal buoyancy force, chemical reaction, time and angle of inclination of magnetic field tend to enhance skin friction whereas thermal radiation and solutal buoyancy force have reverse effect on it. It is interesting to note from Table 1 that there exists a separation of flow at the plate on increasing $G_{c}$.

Numerical values of Sherwood number $S_{h}$ are computed from (28) and are presented in Table 3 for various values of $S_{c}, \gamma$ and $t$. It is perceived from Table 3 that $S_{h}$ increases on increasing either $S_{c}$ or $\gamma$ while it decreases on increasing $t$. This implies that chemical reaction tends to enhance the rate of mass transfer at the plate whereas mass diffusion and time have reverse effect on it.

Numerical values of temperature at the plate are computed from (29) and are displayed in Table 4 for various values of $\delta$ and $t$ taking $P_{r}=0.71$. It is apparent from Table 3 that temperature at the plate increases on increasing $t$ whereas it decreases on increasing $\delta$. This implies that thermal radiation and time have tendency to enhance temperature at the plate.

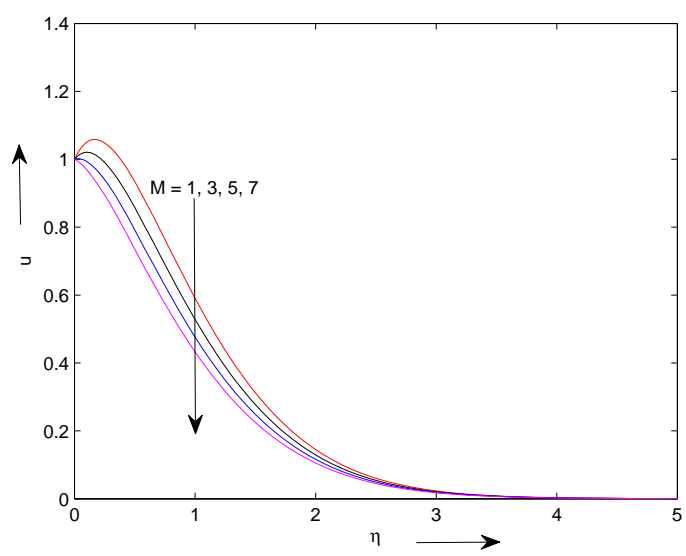

Fig. 2 Velocity profiles when $\delta=0.24, G_{r}=6, G_{c}=8, \gamma=1, \alpha=\pi / 4$ and $t=0.5$. 


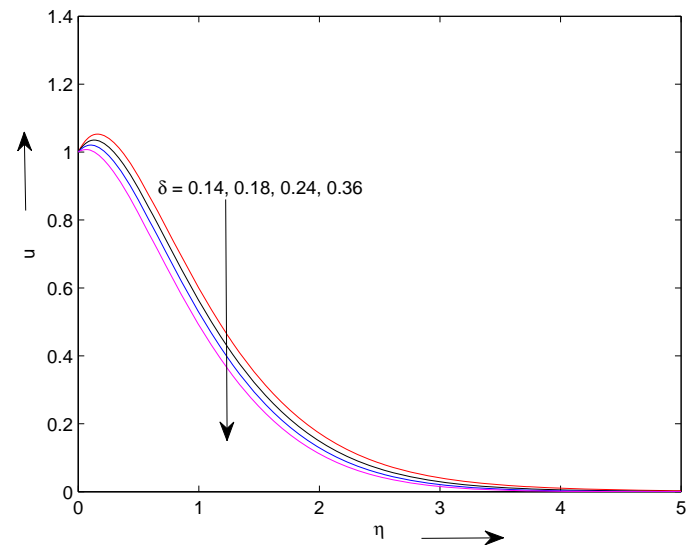

Fig. 3 Velocity profiles when $M=3, G_{r}=6, G_{c}=8, \gamma=1, \alpha=\pi / 4$ and $t=0.5$.

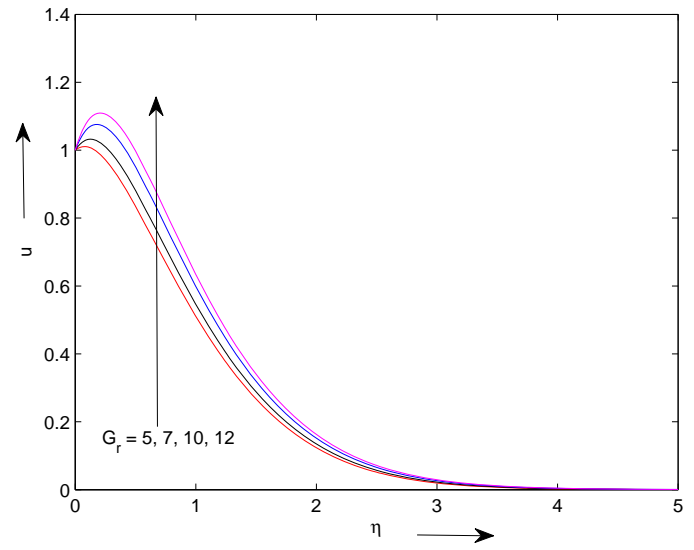

Fig. 4 Velocity profiles when $M=3, \delta=0.24, G_{c}=8, \gamma=1, \alpha=\pi / 4$ and $t=0.5$.

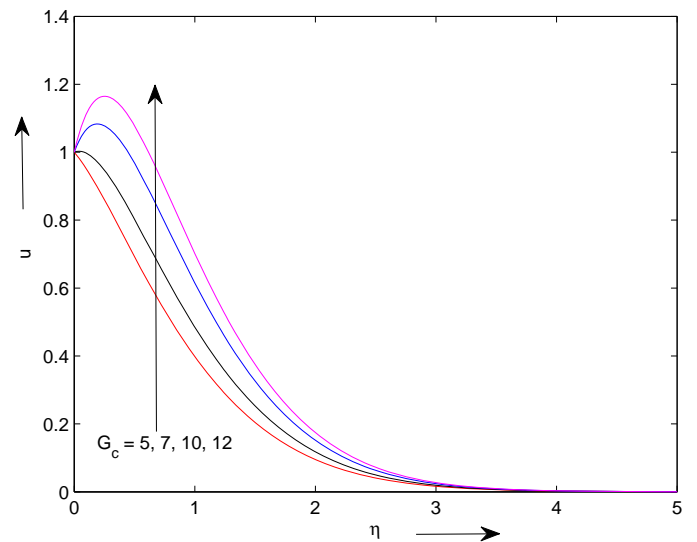

Fig. 5 Velocity profiles when $M=3, \delta=0.24, G_{r}=6, \gamma=1, \alpha=\pi / 4$ and $t=0.5$.

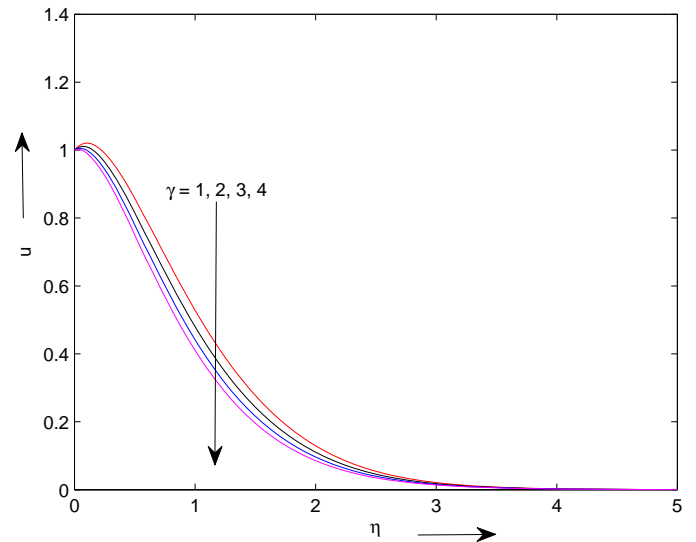

Fig. 6 Velocity profiles when $M=3, \delta=0.24, G_{r}=6, G_{c}=8, \alpha=\pi / 4$ and $t=0.5$.

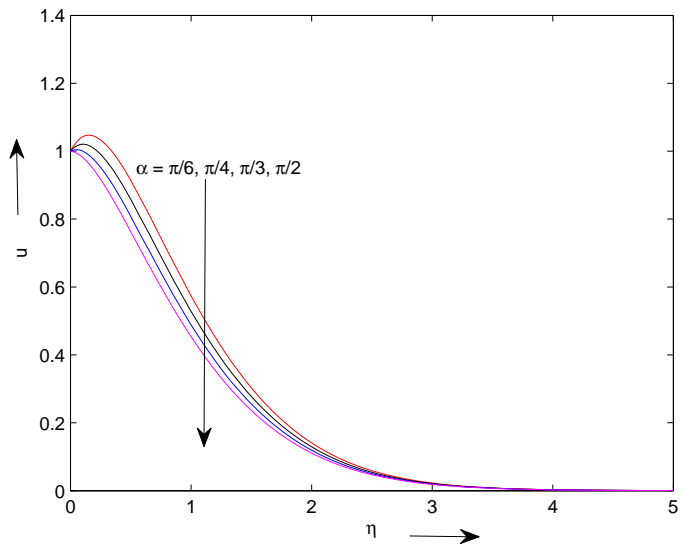

Fig. 7 Velocity profiles when $M=3, \delta=0.24, G_{r}=6, S_{c}=0.6, \gamma=1$ and $t=0.5$.

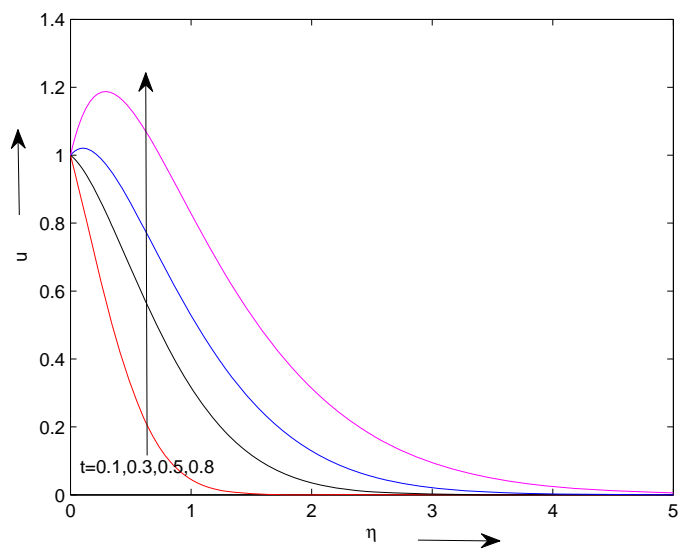

Fig. 8 Velocity profiles when $M=3, \delta=0.24, G_{r}=6, G_{c}=8, \gamma=1$ and $\alpha=\pi / 4$. 


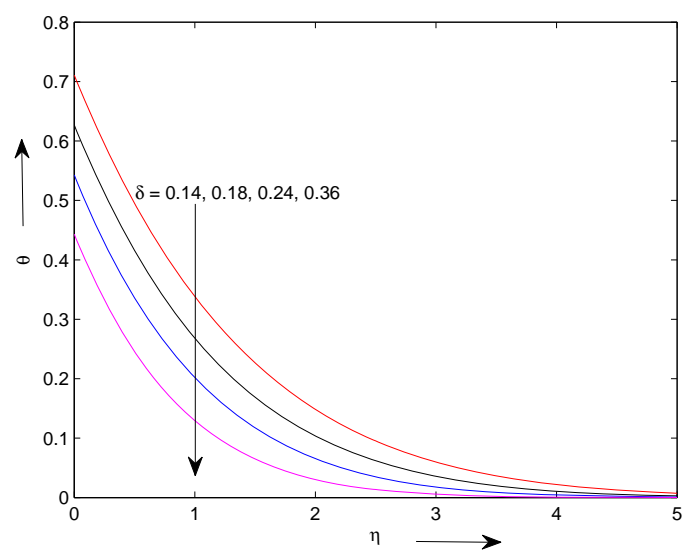

Fig. 9 Temperature profiles when $t=0.5$.

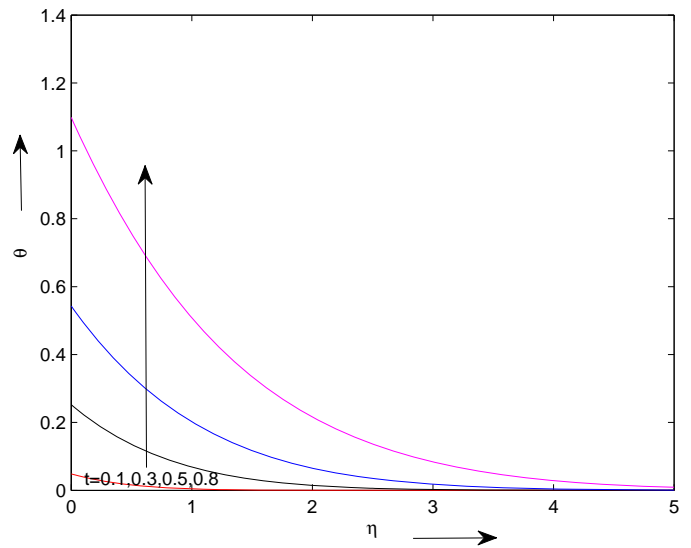

Fig. 10 Temperature profiles when $\delta=0.24$.

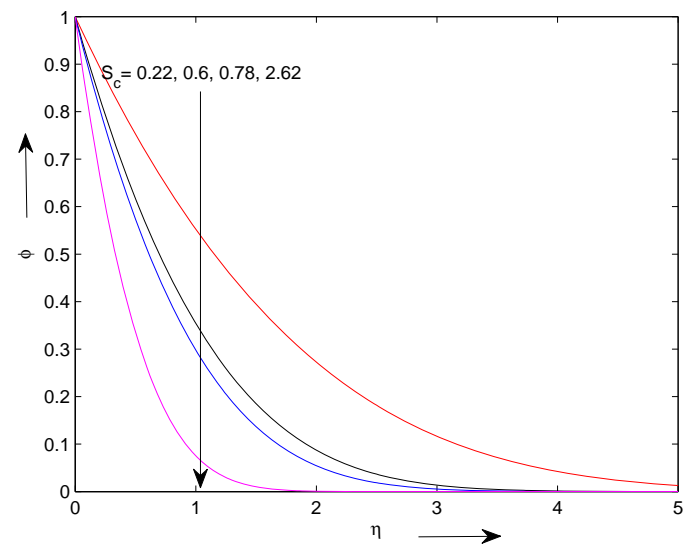

Fig. 11 Concentration profiles when $\gamma=1$ and $t=0.5$.

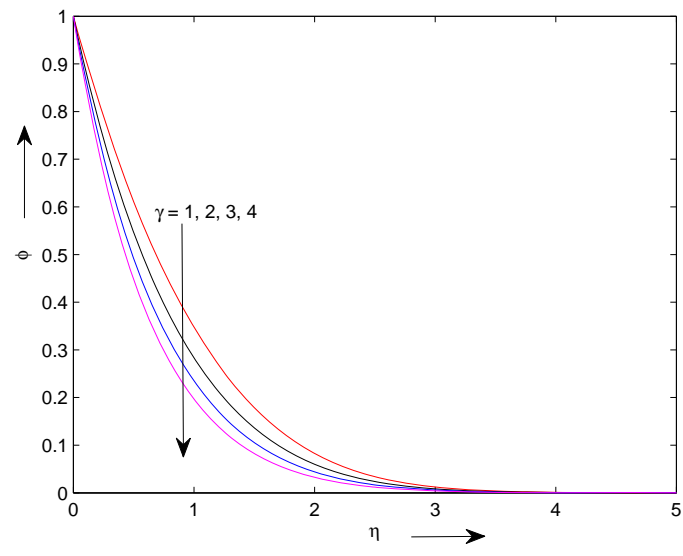

Fig. 12 Concentration profiles when $S_{c}=0.6$ and $t=0.5$.

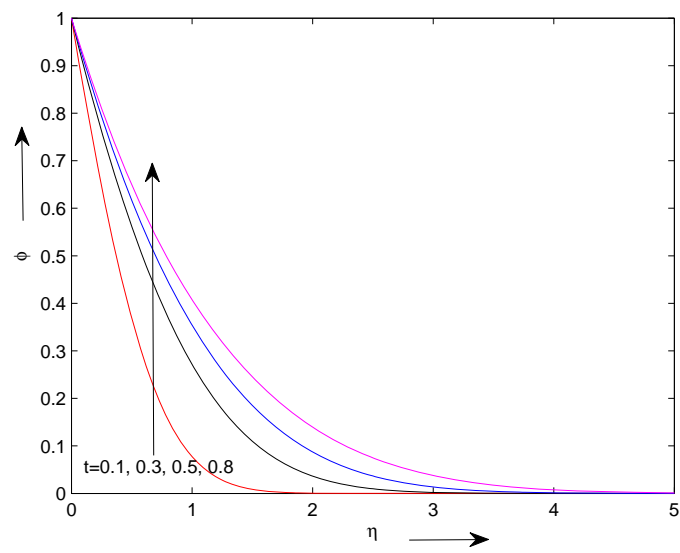

Fig. 13 Concentration profiles when $S_{c}=0.6$ and $\gamma=1$.

Table1 Skin friction $\tau$ when $\gamma=1, \alpha=\pi / 4$ and $t=0.5$.

\begin{tabular}{|l|l|l|l|l|}
\hline$M$ & $\delta$ & $G_{r}$ & $G_{c}$ & $\tau$ \\
\hline $\mathbf{1}$ & 0.24 & 6 & 8 & 0.422743 \\
\hline $\mathbf{3}$ & 0.24 & 6 & 8 & 0.847619 \\
\hline $\mathbf{5}$ & 0.24 & 6 & 8 & 1.22619 \\
\hline 3 & $\mathbf{0 . 1 8}$ & 6 & 8 & 0.568597 \\
\hline 3 & $\mathbf{0 . 2 4}$ & 6 & 8 & 0.847619 \\
\hline 3 & $\mathbf{0 . 3 6}$ & 6 & 8 & 1.360730 \\
\hline 3 & 0.24 & $\mathbf{5}$ & 8 & 0.766275 \\
\hline 3 & 0.24 & $\mathbf{7}$ & 8 & 0.928964 \\
\hline 3 & 0.24 & $\mathbf{1 0}$ & 8 & 1.173000 \\
\hline 3 & 0.24 & 6 & $\mathbf{7}$ & 1.121790 \\
\hline 3 & 0.24 & 6 & $\mathbf{1 0}$ & 0.299271 \\
\hline 3 & 0.24 & 6 & $\mathbf{1 2}$ & -0.249077 \\
\hline
\end{tabular}

Table 2 Skin friction $\tau$ when $M=3, \delta=0.24, G_{r}=6$ and $G_{c}=8$.

\begin{tabular}{|l|l|l|l|}
\hline$\gamma$ & $\alpha$ & $t$ & $\tau$ \\
\hline $\mathbf{1}$ & $\pi / 4$ & 0.5 & 0.847619 \\
\hline $\mathbf{2}$ & $\pi / 4$ & 0.5 & 0.958804 \\
\hline $\mathbf{3}$ & $\pi / 4$ & 0.5 & 1.052760 \\
\hline 1 & $\boldsymbol{\pi} / \mathbf{6}$ & 0.5 & 0.533900 \\
\hline 1 & $\boldsymbol{\pi} / \mathbf{4}$ & 0.5 & 0.847619 \\
\hline 1 & $\boldsymbol{\pi} / \mathbf{3}$ & 0.5 & 1.135350 \\
\hline 1 & $\pi / 4$ & $\mathbf{0 . 3}$ & 0.627134 \\
\hline 1 & $\pi / 4$ & $\mathbf{0 . 5}$ & 0.847619 \\
\hline 1 & $\pi / 4$ & $\mathbf{0 . 8}$ & 2.264950 \\
\hline
\end{tabular}


Table 3 Sherwood number $S_{h}$

\begin{tabular}{|l|l|l|l|}
\hline$S_{c}$ & $\gamma$ & $t$ & $S_{h}$ \\
\hline $\mathbf{0 . 2 2}$ & 1 & 0.5 & 0.5471 \\
\hline $\mathbf{0 . 6 0}$ & 1 & 0.5 & 0.9036 \\
\hline $\mathbf{0 . 7 8}$ & 1 & 0.5 & 1.0303 \\
\hline 0.60 & $\mathbf{1}$ & 0.5 & 0.9036 \\
\hline 0.60 & $\mathbf{2}$ & 0.5 & 1.1505 \\
\hline 0.60 & $\mathbf{3}$ & 0.5 & 1.3678 \\
\hline 0.60 & 1 & $\mathbf{0 . 3}$ & 1.0259 \\
\hline 0.60 & 1 & $\mathbf{0 . 5}$ & 0.9036 \\
\hline 0.60 & 1 & $\mathbf{0 . 8}$ & 0.8346 \\
\hline
\end{tabular}

Table 4 Plate temperature $\theta(0, t)$

\begin{tabular}{|l|l|l|}
\hline$\delta$ & $t$ & $\theta(0, t)$ \\
\hline $\mathbf{0 . 1 8}$ & 0.5 & 0.626877 \\
\hline $\mathbf{0 . 2 4}$ & 0.5 & 0.542892 \\
\hline $\mathbf{0 . 3 6}$ & 0.5 & 0.443269 \\
\hline 0.24 & $\mathbf{0 . 3}$ & 0.252313 \\
\hline 0.24 & $\mathbf{0 . 5}$ & 0.542892 \\
\hline 0.24 & $\mathbf{0 . 8}$ & 1.098740 \\
\hline
\end{tabular}

\section{CONCLUSIONS}

The present study analyses unsteady hydromagnetic free convection heat and mass transfer flow of a viscous, incompressible, electrically conducting and optically thick heat radiating fluid past an impulsively moving infinite vertical plate with ramped heat flux embedded in a fluid saturated porous medium in the presence of chemical reaction and inclined magnetic field. Significant results are summarized below:

1) The effects of thermal radiation, thermal buoyancy force, solutal buoyancy force and time on fluid velocity are opposite to those of magnetic field, chemical reaction and angle of inclination of magnetic field.

2) By changing the angle of inclination of magnetic field, fluid flow may be accelerated / retarded which may have bearings on the problems of practical interest.

3) Thermal radiation and time tend to enhance the fluid temperature.

4) There is a steady drop in concentration level of the fluid for high mass diffusion and chemical reaction whereas time has a reverse effect on it.

5) Magnetic field, thermal buoyancy force, chemical reaction, angle of inclination of magnetic field and time tend to enhance the skin friction whereas thermal radiation and solutal buoyancy force have reverse effect on it.

6) There exists a separation of flow at the plate on increasing the strength of solutal buoyancy force.

7) Chemical reaction has a tendency to enhance the rate of mass transfer whereas mass diffusion and time have reverse effect on it.

8) Thermal radiation and time tend to enhance the plate temperature.

\section{ACKNOWLEDGEMENT}

Authors are grateful to the reviewer for his valuable suggestions and comments which helped them to improve the quality of this research paper. One of the authors P. K Mandal, is thankful to IIT (ISM) Dhanbad to provide research fellowship to carry out this research work.

\section{NOMENCLATURE}

$\begin{array}{ll}\vec{B} & \text { magnetic field vector } \\ B_{0} & \text { uniform magnetic field } \\ C & \text { species concentration } \\ C_{p} & \text { specific heat at constant pressure } \\ D_{m} & \text { mass diffusivity } \\ \vec{E} & \text { electric field vector } \\ g & \text { acceleration due to gravity } \\ G_{c} & \text { solutal Grashof number } \\ G_{r} & \text { thermal Grashof number } \\ k & \text { thermal conductivity } \\ k_{1} & \text { permeability of the medium } \\ k_{2} & \text { chemical reaction coefficient } \\ k^{*} & \text { Rosseland mean absorption coefficient } \\ K_{1} & \text { permeability parameter } \\ M & \text { Magnetic parameter } \\ P_{r} & \text { Prandtl number } \\ q & \text { constant heat flux } \\ q_{r} & \text { radiative heat flux } \\ R & \text { radiation parameter } \\ S_{c} & \text { Schmidt number } \\ T & \text { fluid temperature } \\ t_{0} & \text { characteristic time } \\ t^{\prime} & \text { time } \\ t & \text { dimensionless time } \\ u^{\prime} & \text { fluid velocity } \\ u & \text { dimensionless fluid velocity } \\ U_{0} & \text { reference velocity } \\ & \end{array}$

\section{Greek Symbols}

$\alpha \quad$ angle of inclination of magnetic field

$\beta \quad$ thermal expansion coefficient

$\beta^{*} \quad$ concentration expansion coefficient

$\gamma \quad$ chemical reaction parameter

$\delta \quad$ effective Prandtl number

$v \quad$ kinematic coefficient of viscosity

$\rho \quad$ fluid density

$\sigma \quad$ electrical conductivity

$\sigma^{*} \quad$ Stefan-Boltzman constant

\section{Subscripts}

$w \quad$ at the plate

$\infty \quad$ outside the boundary layer 


\section{REFERENCES}

Aboeldahab, E. M. and Azzam, G. E. A., 2005, “Thermal Radiation Effects on Magnetohydrodynamic Flow Past a Semi-Infinite Vertical Plate in the Presence of Mass Diffusion," Canadian Journal of Physics, 83(3), 243-256.

http://dx.doi.org/10.1139/p04-084

Ahmed, S. and Kalita, K., 2013, "Analytical and Numerical Study for MHD Radiating Flow over an Infinite Vertical Surface Bounded by a Porous Medium in Presence of Chemical Reaction," Journal of Applied Fluid Mechanics, 6(4), 597-607.

Akram, S., Nadeem, S. and Hussain, A., 2014, "Effects of Heat and Mass Transfer on Peristaltic Flow of a Bingham Fluid in the Presence of Inclined Magnetic Field and Channel with Different Wave Forms," Journal of Magnetism and Magnetic Materials, 362,184-192. http://dx.doi.org/10.1016/j.jmmm.2014.02.063

Azzam, G. E. A., 2002, "Radiation Effects on the MHD Mixed Free Forced Convective Flow Past a Semi-Infinite Moving Vertical Plate for High Temperature Differences," Physica Scripta, 66(1), 71. http://dx.doi.org/10.1238/Physica.Regular.066a00071

Beg, O. A., Ghosh, S. K. and Narahari, M., 2010, "Mathematical Modeling of Oscillatory MHD Couette Flow in a Rotating Highly Permeable Medium Permeated by an Oblique Magnetic Field," Chemical Engineering Communications, 198(2), 235-254.

http://dx.doi.org/10.1080/00986445.2010.500165

Chambre, P. L. and Young, J. D., 1958, "On the Diffusion of a Chemically Reactive Species in a Laminar Boundary Layer Flow," Physics of Fluids, 1(1), 48.

http://dx.doi.org/10.1063/1.1724336

Chamkha, A. J., Rashad, A. M. and Mudhaf, H. A., 2012, "Heat and Mass Transfer from Truncated Cones with Variable Wall Temperature and Concentration in the Presence of Chemical Reaction Effects," International Journal of Numerical Methods for Heat and Fluid flow, 22(3), 357-376.

http://dx.doi.org/10.1108/09615531211208060

Chandran, P., Sacheti, N. C. and Singh, A. K., 1998, "Unsteady Hydromagnetic Free Convection Flow with Heat Flux and Accelerated Boundary Motion," Journal of the Physical Society of Japan, 67(1), 124129.

http://dx.doi.org/10.1143/JPSJ.67.124

Chen, S. L., Ma, H. K. and Chen, D. Y., 1993, "Radiation Blockage by the Interaction of Thermal Radiation with Conduction and Convection in the Combustion of the Condensed Fuel," International Communications in Heat and Mass Transfer, 20(1), 145-147.

http://dx.doi.org/10.1016/0735-1933(93)90015-N

Chaudhary, R. C. and Jain, A., 2007, "Combined Heat and Mass Transfer Effects on MHD Free Convection Flow Past an Oscillating Plate Embedded in a Porous Medium," Romanian Journal of Physics, 52(5-7), 505-524.

Cogley, A. C., Gilles, S. E. and Vincenti, W. G., 1968, "Differential Approximation for Radiative Transfer in a Non-Grey Gas Near Equilibrium," AIAA Journal, 6(3), 551-553.

http://dx.doi.org/10.2514/3.4538
Eckert, E. R. and Drake, R. M., 1972, Analysis of Heat and Mass Transfer, Mc-Graw Hill, New York.

Eldabe, N. T. M., Elbashbeshy, E. M. A., Hasanin, W. S. A. and Elsaid, E. M., 2011, "Unsteady Motion of MHD Viscous Incompressible Fluid with Heat and Mass Transfer through Porous Medium Near a Moving Vertical Plate," International Journal of Energy and Technology, 3, 111 .

El-Kabeir, S. M. M., Modather, M. and Rashad, A. M., 2015, "Heat and Mass Transfer by Unsteady Natural Convection over a Moving Vertical Plate Embedded in a Saturated Porous Medium with Chemical Reaction, Soret and Dufour Effects," Journal of Applied Fluid Mechanics, 8(3), 453-463.

Fairbanks, D. F. and Wilke, C. R., 1950, "Diffusion and Chemical Reaction in an Isothermal Laminar Flow along a Soluble Flat Plate," Industrial and Engineering Chemistry Research, 42(3), 471-475. http://dx.doi.org/10.1021/ie50483a022

Gedda, H., Powell, J., Wahlstrom, G., Li, W. B., Engstrom, H. and Magnusson, C., 2002, "Energy Redistribution during $\mathrm{CO}_{2}$ Laser Cladding," Journal of Laser Applications, 14(2), 78-82. http://dx.doi.org/10.2351/1.1471565

Ghosh, S. K., Bhattacharjee, P. K., 2000, "Hall Effects on Steady Hydromagnetic Flow in a Rotating Channel in the Presence of an Inclined Magnetic Field," Czechoslovak Journal of Physics, 50(6), 759767.

http://dx.doi.org/10.1023/A:1022839020051

Ghosh, S. K., Das, S. and Jana, R. N., 2015, "Transient MHD Free Convective Flow of an Optically Thick Gray Gas Past a Moving Vertical Plate in the Presence of Thermal Radiation and Mass Diffusion," Journal of Applied Fluid Mechanics, 8(1), 65-73.

Hayday, A. A., Bowlus, D. A. and McGraw, R. A., 1967, "Free Convection from a Vertical Plate with Step Discontinuous in Surface Temperature," Journal of Heat Transfer, 89(3), 244-249.

http://dx.doi.org/10.1115/1.3614371

Hossain, M. A. and Mandal, A. C., 1985, "Mass Transfer Effects on the Unsteady Hydromagnetic Free Convection Flow Past an Accelerated Vertical Porous Plate," Journal of Physics D: Applied Physics, 18 (7), L63.

http://dx.doi.org/10.1088/0022-3727/18/7/003

Hussanan, A., Ismail, Z., Khan, I., Hussein, A. G. and Shafie, S., 2014, "Unsteady Boundary Layer MHD Free Convection Flow in a Porous Medium with Constant Mass Diffusion and Newtonian Heating," European Physical Journal Plus, 129(3), 46.

http://dx.doi.org/10.1140/epjp/i2014-14046-x

Ibrahim, F. S., Hassanien, I. A. and Bakr, A. A., 2004, "Unsteady Magnetohydrodynamic Micropolar Fluid Flow and Heat Transfer over a Vertical Porous Medium in the Presence of Thermal and Mass Diffusion with Constant Heat Source," Canadian Journal of Physics, 82(10), 775790.

http://dx.doi.org/10.1139/p04-021

Incropera, F. P., Bergman, T. L., Lavine, A. S. and Dewitt, D. P., 2011, Fundamentals of Heat and Mass Transfer, John Willey \& Sons, New York. 
Jha, B. K., 1991, "MHD Free-Convection and Mass-Transform Flow through a Porous Medium," Astrophysics and Space Science, 175(2), 283-289.

http://dx.doi.org/10.1007/BF00644290

Kao, T. T., 1975, "Laminar Free Convective Heat Transfer Response along a Vertical Flat Plate with Step Jump in Surface Temperature," Letters in Heat and Mass Transfer, 2(5), 419-428.

http://dx.doi.org/10.1016/0094-4548(75)90008-9

Kays, W. M. and Crawford, M. E., 1993, Convective Heat and Mass Transfer, $3^{\text {rd }}$ ed., Mc-Graw Hill, New York.

Kelleher, M., 1971, "Free Convection from a Vertical Plate with Discontinuous Wall Temperature," Journal of Heat Transfer, 93(4), 349356.

http://dx.doi.org/10.1115/1.3449830

Khalid, A., Khan, I. and Shafie, S., 2015, "Exact Solutions for Free Convection Flow of Nanofluids with Ramped Wall Temperature," European Physical Journal Plus, 130(4), 57-59.

http://dx.doi.org/10.1140/epjp/i2015-15057-9

Lee, S., and Yovanovich, M. M., 1991, "Laminar Natural Convection from a Vertical Plate with a Step Change in Wall Temperature," Journal of Heat Transfer, 113, 501-504.

http://dx.doi.org/10.1115/1.2910591

Makinde, O. D. and Sibanda, P., 2008, "Magnetohydrodynamic Mixed Convection Flow and Heat and Mass Transfer Past a Vertical Plate in a Porous Medium with Constant Wall Suction," Journal of Heat Transfer 130(11), 112602.

http://dx.doi.org/10.1115/1.2955471

Mbeledogu, I. U. and Ogulu, A., 2007, "Heat and Mass Transfer of an Unsteady MHD Natural Convection Flow of a Rotating Fluid Past a Vertical Porous Flat Plate in the Presence of Radiative Heat Transfer," International Journal of Heat and Mass Transfer, 50(9), 1902-1908. http://dx.doi.org/10.1016/j.ijheatmasstransfer.2006.10.016

Modather, M., Rashad, A. M. and Chamkha, A. J., 2009, “An Analytical Study on MHD Heat and Mass Transfer Oscillatory Flow of Micropolar Fluid over a Vertical Permeable Plate in a Porous Medium," Turkis Journal of Engineering and Environmental Sciences, 33(4), 245-257.

Muthucumaraswamy, R., Dhanasekar, N. and Prasad, G. E., 2013, "Rotation Effects on Unsteady Flow Past an Accelerated Isothermal Vertical Plate with Variable Mass Transfer in the Presence of Chemical Reaction of First Order," Journal of Applied Fluid Mechanics, 6 (4), 485490.

Nandkeolyar, R. and Das, M., 2015, "MHD Free Convective Radiative Flow Past a Flat Plate with Ramped Temperature in the Presence of inclined Magnetic Field," Computational and Applied Mathematics, 34(1), 109-123.

http://dx.doi.org/10.1007/s40314-013-0107-6

Nasab, S. A. G. and Maramisaran, M., 2009, "Transient Numerical Analysis of a Multi Layered Porous Heat Exchanger Including Gas Radiation Effects," International Journal of Thermal Sciences, 48(8), 1586-1595.

http://dx.doi.org/10.1016/j.ijthermalsci.2008.12.014

Nield, D. A. and Bejan, A., 2006, Convection in Porous Media, SpringerVerlag, New York.
Obidina, S. P. and Kiseleva, M. N., 1980, "Production of Glass with High Radiation Absorption in the Range 0.9-1.2 $\mu \mathrm{m}$," Glass and Ceramics, 37(8), 376-378.

http://dx.doi.org/10.1007/BF00703936

Palani, G. and Abbas, I. A., 2009, "Free Convection MHD Flow with Thermal Radiation from an Impulsively Started Vertical Plate," Nonlinear Analysis: Modelling and Control, 14(1), 73-84.

Patra, R. R., Das, S., Jana, R. N. and Ghosh, S. K., 2012, “Transient Approach to Radiative Heat Transfer Free Convection Flow with Ramped Wall Temperature," Journal of Applied Fluid Mechanics, 5(9), 239-247.

Prakash, J., Bhanumathi, D., Kumar, A. G. V. and Varma, S. V. K., 2013, "Diffusion-Thermo and Radiation Effects on Unsteady MHD Flow through Porous Medium Past an Impulsively Started Infinite Vertical Plate with Variable Temperature and Mass Diffusion," Transport in Porous Media, 96(1), 135-151.

http://dx.doi.org/10.1007/s11242-012-0078-x

Raju, M. C., Varma, S. V. K. and Seshaiah, B., 2015, "Heat Transfer Effects on a Viscous Dissipative Fluid Flow Past a Vertical Plate in the Presence of Induced Magnetic Field," Ain Shams Engineering Journal, 6(1), 333-339.

http://dx.doi.org/10.1016/j.asej.2014.07.009

Raptis, A. and Massalas, C. V., 1998, "Magnetohydrodynamic Flow Past a Plate by the Presence of Radiation," Heat and Mass Transfer, 34(2-3), 107-109.

http://dx.doi.org/10.1007/s002310050237

Raptis, A., Perdikis, C. and Takhar, H. S., 2004, "Effect of Thermal Radiation on MHD Flow," Applied Mathematics and Computation, 153(3), 645-649.

http://dx.doi.org/10.1016/S0096-3003(03)00657-X

Rashad, A. M., Modather, M. and Chamkha, A. J., 2011, "MHD Free Convective Heat and Mass Transfer of a Chemically-Reacting Fluid from Radiate Stretching Surface Embedded in a Saturated Porous Medium," International Journal of Chemical Reactor Engineering, 9 (1), 1-15. http://dx.doi.org/10.1515/1542-6580.2501

Reddy, K. S. and Kumar, N. S., 2008, "Combined Laminar Natural Convection and Surface Radiation Heat Transfer in a Modified Cavity Receiver of Solar Parabolic Dish," International Journal of Thermal Sciences, 47(12), 1647-1657.

http://dx.doi.org/10.1016/j.ijthermalsci.2007.12.001

Reddy, N. P., Raju, M. C. and Varma, S. V. K., 2008, "Effect of Aligned Magnetic Field on Unsteady Flow between a Stretching Sheet and Oscillating Porous Plate with Constant Suction," International Review of Pure and Applied Mathematics, 4(2), 211-218.

Saladino, A. J. and Farmer, R. C., 1993, Radiation/Convection Coupling in Rocket Motor and Plume Analysis, Final Report SECA, Inc., Huntsville, Alabama, USA.

Seth, G. S., Hussain, S. M. and Sarkar, S., 2014, "Effects of Hall Current and Rotation on Unsteady MHD Natural Convection Flow with Heat and Mass Transfer Past an Impulsively Moving Vertical Plate in the Presence of Radiation and Chemical Reaction," Bulgarian Chemical Communications, 46(4), 704-718. 
Seth, G. S., Nandkeolyar, R., Ansari, M. S., 2010, "Hartmann Flow in a Rotating System in the Presence of Inclined Magnetic Field with Hall Effects," Tamkang Journal of Science and Engineering, 13(3), 243-252.

Seth, G. S., Nandkeolyar, R., Ansari, M. S., 2013, "Effects of Thermal Radiation and Rotation on Unsteady Hydromagnetic Free Convection Flow Past an Impulsively Moving Vertical Plate with Ramped Temperature in a Porous Medium," Journal of Applied Fluid Mechanics, 6(1), 27-38.

Seth, G. S., Nandkeolyar, R., Mahto, N., Singh, S. K., 2009, "MHD Couette Flow in a Rotating System in the Presence of an Inclined Magnetic Field," Applied Mathematical Science, 3(57-60), 2919-2932.

Siegel, R. and Howell, J. R., 1972, Thermal Radiation Heat Transfer, Student edition, Mac-Graw Hill.
Takhar, H. S., Gorla, R. S. R. and Soundalgekar, V. M., 1996, "Short Communication Radiation Effects on MHD Free Convection Flow of a Gas Past a Semi-Infinite Vertical Plate," International Journal of Numerical Methods for Heat and Fluid Flow, 6(2), 77-83. http://dx.doi.org/10.1108/09615539610113118

Umamaheswar, M., Raju, M. C. and Varma, S. V. K., 2015a, "Analysis of MHD Transient Free Convection Flow of a Newtonian Fluid Past an Infinite Vertical Porous Plate," Frontiers in Heat and Mass Transfer, $6(18)$.

http://dx.doi.org/10.5098/hmt.6.18

Umamaheswar, M., Varma, S. V. K., Raju, M. C. and Chamkha, A. J., 2015b, "Unsteady MHD Free Convective Double Diffusive ViscoElastic Fluid Flow Past an Inclined Permeable Plate in the Presence of Viscous Dissipation and Heat Absorption," Special topics and Reviews in Porous Media: An International Journal, 6(4), 333-342. http://dx.doi.org/10.1615/SpecialTopicsRevPorousMedia.v6.i4.20 